J. Lake Sci. (湖泊科学) , 2017, 29(1): 105-115

DOI 10. 18307/2017. 0112

(c) 2017 by Journal of Lake Sciences

\title{
几种水陆交错带植物对湖滨带底质的稳固作用”
}

\author{
姚 程 $^{1}$, 胡小贞 ${ }^{2 * *}$, 耿荣妹 $^{2}$, 薛彦君 $^{2}$, 成小英 ${ }^{3}$ \\ ( 1 : 南京工业大学城市建设学院, 南京 210000) \\ (2: 中国环境科学研究院湖泊生态环境创新基地/国家环境保护湖泊污染控制重点实验室,环境基准与风险评估国家重 \\ 点实验室, 北京 100012) \\ (3: 江南大学环境学院,无锡 214000)
}

\begin{abstract}
摘 要: 为了研究几种常见水陆交错带植物对底质稳固性的影响, 选取太湖贡湖湾水陆交错带内的双穗雀稗 (Paspalum distichum)、李氏禾 (Leersia hexandra)、香菇草 (Hydrocotyle vulgaris)、黄花水龙 (Ludwigia peploides) 和黄菖蒲 (Iris pseudacorus $) 5$ 种水生/湿生植物植物, 并利用长江下游常见沙壤土和湖滨带新生底质两种土壤, 开展了 5 种植物对底质稳 固作用的室内研究. 结果表明底质孔隙度减少、细小粒径 $(<50 \mu \mathrm{m})$ 增加有利于底质稳固, 改善上覆水指标, 减少扰动给 上覆水所带来的悬浮颗粒物. 直径 $\leqslant 1 \mathrm{~mm}$ 的须根量、须根长度和须根面积与底质孔隙度和粒径分布增益值之间存在线 性回归关系, 双穗雀稗、李氏禾、香菇草的根系参数与增益值之间存在斜率为 $0.006 \sim 1.727$ 的线性正相关关系; 黄花水龙、 黄菖蒲植物根系参数与增益值之间则存在斜率为 $-0.091 \sim-0.011$ 的线性负相关关系. 黄菖蒲与黄花水龙的根长密度分别 为 11.495 和 $9.475 \mathrm{~cm} / \mathrm{cm}^{3}$, 根表面积密度分别为 0.368 和 $0.294 \mathrm{~cm}^{2} / \mathrm{cm}^{3}$, 根重密度分别为 1.844 和 $0.944 \mathrm{mg} / \mathrm{cm}^{3}$, 两种植 物对底质孔隙度的增益值分别为 $15 \%$ 和 $9 \%$, 对底质粒径分布的增益值分别为 $92 \%$ 和 $47 \%$; 双穗雀稗、李氏禾、香菇草的 根长密度分别为 $1.057 、 7.368$ 和 $0.651 \mathrm{~cm} / \mathrm{cm}^{3}$, 根表面积密度分别为 $0.033 、 0.228$ 和 $0.022 \mathrm{~cm}^{2} / \mathrm{cm}^{3}$, 根重密度分别为 0 . $678 、 2.537$ 和 $0.160 \mathrm{mg} / \mathrm{cm}^{3}, 3$ 种植物根系参数对底质孔隙度的增益值分别为 $6 \%$ 、36\%和 $1 \%, 3$ 种植物根系参数对底质粒 径分布的增益值分别为 $16 \% 、 17 \%$ 和 $-13 \% .5$ 种植物通过根系提高底质的稳定性, 减少底质在水力扰动下悬浮物质以及 营养盐的释放, 从效能上表现为李氏禾>双穗雀稗>黄菖蒲 > 黄花水龙>香菇草.
\end{abstract}

关键词: 植物须根; 底质稳固性; 底质粒径分布; 底质孔隙度; 根系参数

\section{Effects of five literal-zone plants on lakeshore sediment stabilization}

\author{
YAO Cheng $^{1}$, HU Xiaozhen ${ }^{2 * *}$, GENG Rongmei ${ }^{2}$, XUE Yanjun $^{2}$ \& CHENG Xiaoying ${ }^{3}$ \\ (1: College of Urban Construction, Nanjing Tech University, Nanjing 210000, P.R.China) \\ (2: State Environmental Protection Key Laboratory for Lake Pollution Control, Research Center of Lake Eco-Environment, \\ State Key Laboratory of Environmental Criteria and Risk Assessment, Chinese Research Academy of Environmental Sci- \\ ences, Beijing 100012, P.R.China) \\ (3: Environment College, Jiangnan University, Wuxi 214000 , P.R.China)
}

\begin{abstract}
Small-scale physical experiment in the laboratory has been taken for simulating the effects of Paspalum distichum, Leersia hexandra, Hydrocotyle vulgaris, Ludwigia peploides and Iris pseudacorus on the literal-zone sediment stabilization by collecting sandy soil from lower reaches of Yangtze River and clay from the literal-zone. The results indicated that the reduced degree of porosity and increased proportion of specified particle ( diameter $<50 \mu \mathrm{m}$ ) of sediments could improve the quality of overlying water. The linear regression model of the improvement of sediment stabilization with five kinds of plant (Paspalum distichum, Leersia hexandra, Hydrocotyle vulgaris, Ludwigia peploides and Iris pseudacorus and fine root parameters (root length density, root surface area density and root weight density) is put forward. The correlations of root parameters of Leersia hexandra, Paspalum distichum, Hydrocotyle vulgaris with the degree of sediment porosity and with the particle diameter have positively linear correlations with slop
\end{abstract}

* 国家水体污染控制与治理科技重大专项( 2013ZX07101-014-004, 2014ZX07510-001-01) 资助. 2016-03-09 收稿; 2016-05-10 收修改稿. 姚程( 1990 ), 男, 硕士研究生; E-mail: yao948416159@163.com.

** 通信作者;E-mail:huxz@ craes.org.cn. 
arranging from 0.006 to 1.727 , respectively, while the negative linear correlation of Iris pseudacorus, Ludwigia peploides with slop arranging from -0.091 to -0.011 . Improvements of sediment porosity of Iris pseudacorus and Ludwigia peploides were attained to $15 \%$ and $9 \%$, respectively, and improvement of particle diameters to $92 \%$ and $47 \%$, respectively. The linear equation was used to calculate the plants' root parameter: 11.495 and $9.475 \mathrm{~cm} / \mathrm{cm}^{3}$ in root length density, 0.368 and $0.294 \mathrm{~cm}^{2} / \mathrm{cm}^{3}$ in root surface area density, 1.844 and $0.944 \mathrm{mg} / \mathrm{cm}^{3}$ in root weight density, for root parameters with sediment porosity and with the particle diameter, respectively. The sediment porosity improvements of Leersia hexandra, Paspalum distichum and Hydrocotyle vulgaris were attained to $6 \%, 36 \%$ and $1 \%$, and distribution of particle diameter improvement to $16 \%, 17 \%$ and $-13 \%$, according to linear equation which was calculated with plants' root parameter: $1.057,7.368$ and $0.651 \mathrm{~cm} / \mathrm{cm}^{3}$ in root length density, $0.033,0.228$ and $0.022 \mathrm{~cm}^{2} / \mathrm{cm}^{3}$ in root surface area density, and $0.678,2.537$ and $0.160 \mathrm{mg} / \mathrm{cm}^{3}$ in root weight density, respectively. Five literalzone plants are all proved to improve sediment stabilization, which repress eutrophication and sediment resuspension caused by soil erosion on lakeshore. Comparably, the effectiveness from high to low are in descending order from Leersia hexandra, Paspalum distichum, Iris pseudacorus, Ludwigia peploides to Hydrocotyle vulgaris.

Keywords: Plant fine root; sediment stabilization; distribution of particle diameter; sediment porosity ; root parameter

水陆交错带是湖泊水生态系统与陆地生态系统之间非常重要的过渡带, 具有拦截地表径流、提供生物 栖息地、改善湖滨景观等任务 ${ }^{[1]}$. 底质是水陆交错带生境的重要因素,合适、稳定的底质可以为水陆交错带 植物生长提供良好的立地条件, 不同的植物生长反过来也会对底质尤其是新生底质的理化特征产生较大影 响, 从而推动底质发生演变 ${ }^{[2]}$. 研究者从植物对土壤修复改善作用出发, 指出发达的植物根系不仅仅起到为 植物提供水分、养分的功能, 还利用自身在土体中穿插、缠绕、固结等作用强化土体的抗侵蚀能力 ${ }^{[3]}$. 吴彦等 就对乔木类植物根系对土壤抗侵蚀性的影响做过系统的研究, 指出乔木类植物须根量直接影响着土壤的有 机质、孔隙度等理化参数, 须根量越大越有利于土壤理化性质的改变 ${ }^{[4]}$. 近年来, 随着对湖泊生态环境的深 人研究, 研究者发现水陆交错带植物可以有效减少底质悬浮. 如 Horppila 等就通过对照实验指出了浅水湖 泊中几种浮叶、沉水、挺水群落植物具有抑制底质中磷的释放, 增强底质抗悬浮的作用 ${ }^{[5]}$. Charles 通过对比 南达科他州沙湖 (Sand Lake) 中开阔水域受风浪影响的底质悬浮情况与挺水植物区域的悬浮情况, 发现开阔 区比挺水植物区严重得多, 证明了挺水植物对增强湖泊底质抗悬浮能力的重要意义 ${ }^{[6]}$. Horppila 等还在芬兰 南部希登韦西湖 (Lake Hiidenvesi) 通过野外监测与室内模拟实验进行对比, 发现金鱼藻 (Ceratophyllum demersum)、钝叶眼子菜 (Potamogeton obtusifolius) 等沉水植物可以有效减少底质在水体扰动条件下悬浮颗粒物 的释放 ${ }^{[7]}$. 水生植物被证明可以有效克制水陆交错带底质在风浪扰动条件下的悬浮上扬, 但目前的研究还 主要处于这种作用的发觉阶段, 尚未深人到底质内部结构变化, 缺少植物与这种变化之间的量化研究. 本文 通过室内模拟实验研究几种水陆交错带植物对底质稳固性能的影响, 量化植物与底质稳固性之间的关系, 为湖滨带水生植物群落构建中植物物种的选取以及栽种密度的确定提供参考.

\section{1 材料与方法}

\section{1 植物与底质来源}

实验所用的植物和底质来自于无锡贡湖湾太湖新城贡湖生态修复示范工程区 $\left(31^{\circ} 27^{\prime} 19.91^{\prime \prime} \sim 31^{\circ} 27^{\prime} 25.38^{\prime \prime} \mathrm{N}\right.$, $120^{\circ} 19^{\prime} 48.04^{\prime \prime} \sim 120^{\circ} 19^{\prime} 56.39^{\prime \prime} \mathrm{E}$ ), 该示范区原为鱼塘, 2013 年退渔还湖后经过基底修复改善, 于 2014 年 5 月 经人工植被修复后形成水陆交错带示范区. 示范区内底质主要为外来覆土, 属于湖滨带生态修复区域新生 底质, 其孔隙度为 $37 \%$, 含水率为 $24 \%$, 粒径分布以 $100 \mu \mathrm{m}$ 以下的细小颗粒为主. 实验中还采集生态修复示 范区外的沙壤底质作为实验对象, 其孔隙度为 $45 \%$, 含水率为 $25 \%$, 粒径分布以 $100 \mu \mathrm{m}$ 以上的中细、中粗颗 粒为主.

实验中水陆交错带植物均为长江中下游常见种类, 于 2014 年 5 月人工种植后生长稳定并形成群落. 采 集示范区分布面积大、生长良好的 5 种水生植物作为实验对象, 包括双穗雀稗 (Paspalum distichum)、李氏禾 (Leersia hexandra)、香菇草 (Hydrocotyle vulgaris)、黄花水龙 (Ludwigia peploides)、黄菖蒲 (Iris pseudacorus $)^{[8-12]}$.

\section{2 实验设置}

实验装置 (图 1) 经塑料养殖箱改造而成, 每个养殖箱底均匀铺设 $20 \mathrm{~cm}$ 底质. 每种水生植物分别在水陆 
交错带新生底质和沙壤底质两种底质中进行种植, 并设平行样, 同时两种底质均保留空白参考组. 共设置 5 个实验组和 1 个空白对照组, 植物栽种后先少量加水, 保持底质润湿, 等待 $24 \mathrm{~h}$ 观察栽种效果, 确保实验植 物与底质的紧密结合. 注人纯净水, 淹没植物根部至水深 $10 \mathrm{~cm}$ 左右. 经过 1 周的生长适应期后开始实验, 每个装置中放置曝气头, 曝气量为 $25 \mathrm{ml} / \mathrm{s}$, 通过 FP111 便携式流速仪测定装置中上覆水流速为 $1.9 \sim 2.5 \mathrm{~m} / \mathrm{s}$, 水力梯度为 $15 \mathrm{~s}^{-1}$.

\section{3 数据采集与分析}

连续曝气扰动 1 周 $(7 \mathrm{~d})$ 后监测上覆水悬浮颗粒物浓 度 $(\mathrm{SS})$ 、高锰酸盐指数 $\left(\mathrm{COD}_{\mathrm{Mn}}\right)$ 、总氮 $(\mathrm{TN})$ 及总磷 $(\mathrm{TP})$ 浓度等指标. 实验进行至第 2 周 $(14 \mathrm{~d})$ 后停止水力扰动, 静止 $6 \mathrm{~h}$ 后测定上覆水 $\mathrm{SS} 、 \mathrm{COD}_{\mathrm{Mn}}$ 、 $\mathrm{TN}$ 及 $\mathrm{TP}$ 等指标. 第 $14 \mathrm{~d}$ 静止 $6 \mathrm{~h}$ 后再次进行曝气扰动, 持续至实验第 $20 \mathrm{~d}$, 期间隔 $24 \mathrm{~h}(15 \mathrm{~d}) 、 5 \mathrm{~d}(20 \mathrm{~d})$ 对上覆水 $\mathrm{SS} 、 \mathrm{COD}_{\mathrm{Mn}}$ 、TN 及 $\mathrm{TP}$ 等指标进行测定. 实验结束时 (20 d) 取出植物, 测定其 生物量 (植物鲜重), 用水清洗植物根部, 篮选 $<1 \mathrm{~mm}$ 的须 根, 利用 LA-S 根系分析仪测定根长密度、根表面积密度与 根重密度.

利用钿酸盐比色法测定水体 TP 浓度, 碱性过硫酸钾 比色法测定水体 TN 浓度, 同时测定水体 SS 浓度以及

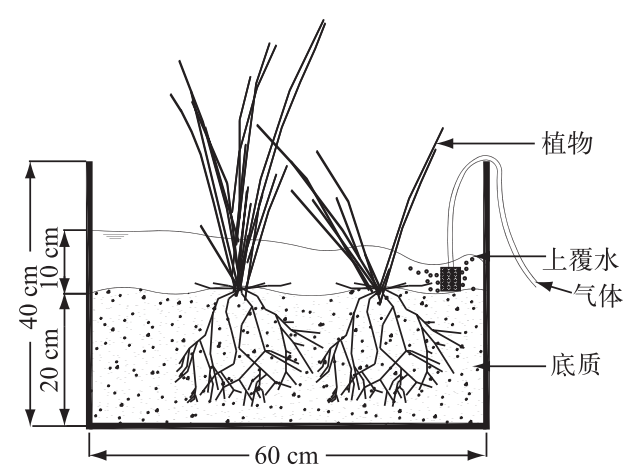

图 1 植物栽种实验装置

Fig.1 Experimental devices of plants $\mathrm{COD}_{\mathrm{Mn}}$. 底质的测定方法参考《沉积物质量调查评估手 册 $\rangle^{[13]}$, 采用凯式定氮仪测定底质 TN 含量, 利用高氯酸-硫酸消解法测定底质 TP 与有效磷含量, 利用重铬 酸钾消解法测定有机质含量, 同时利用容积法测定底质的容重. 本次实验中底质的测定方法参考《沉积物质 量调查评估手册》. 利用激光粒度仪测定底质粒径分布情况. 实验数据利用 Excel、Origin 等软件进行整理, 利 用 SPSS 软件进行相关性分析.

\section{2 结果与讨论}

\section{1 扰动对上覆水指标的影响}

2.1.1 上覆水水质随时间的变化 实验期间各实验组与空白对照组的上覆水 $\mathrm{SS}$ 浓度与 $\mathrm{COD}_{\mathrm{Mn}} 、 \mathrm{TN} 、 \mathrm{TP}$ 浓度 有着不同的变化规律 (图 2). 各实验组 SS 浓度在扰动持续 1 周 (7d) 后达到最高值, 空白对照组 SS 浓度增 幅最大, 由 $70 \mathrm{mg} / \mathrm{L}$ 上升至 $350 \mathrm{mg} / \mathrm{L}$. 水力扰动持续两周后停止扰动并静置 $6 \mathrm{~h}(14 \mathrm{~d}(\mathrm{~S}))$, 各实验组与空白 对照组上覆水 SS 浓度下降至 $150 \mathrm{mg} / \mathrm{L}$ 左右. 静止 $6 \mathrm{~h}$ 后 $(14 \mathrm{~d})$ 各实验组恢复水力扰动, SS 浓度小幅上升. 随着实验进行 $(15 \sim 20 \mathrm{~d}$ ), 各实验组中以新生底质作为实验底质的上覆水 $\mathrm{SS}$ 浓度基本维持在 $120 \mathrm{mg} / \mathrm{L}$, 以 沙壤土作为实验底质的上覆水 SS 浓度存在较大差异, 空白实验组的上覆水 SS 浓度则超过了 $220 \mathrm{mg} / \mathrm{L}$, 远 远高于实验前扰动条件下的水体 SS 浓度.

如图 2 所示, 各实验组与空白对照组上覆水 $\mathrm{COD}_{\mathrm{Mn}} 、 \mathrm{TN} 、 \mathrm{TP}$ 浓度在实验期间持续升高, 各实验组上覆水 $\mathrm{COD}_{\mathrm{Mn}}$ 在实验进行前两周 $(0 \sim 14 \mathrm{~d})$ 由 $5 \mathrm{mg} / \mathrm{L}$ 最高上升至 $9 \mathrm{mg} / \mathrm{L}$, 空白组达到 $7 \mathrm{mg} / \mathrm{L}$, 随后持续扰动 1 周过 程中 $(14 \sim 20 \mathrm{~d})$ 各实验组上覆水 $\mathrm{COD}_{\mathrm{Mn}}$ 下降至 $6.5 \mathrm{mg} / \mathrm{L}$ 以下, 空白组超过 $7 \mathrm{mg} / \mathrm{L}$. 各实验组上覆水 $\mathrm{TN}$ 浓度 在实验进行前两周 $(0 \sim 14 \mathrm{~d})$ 由 $0.35 \mathrm{mg} / \mathrm{L}$ 最高上升至 $0.65 \mathrm{mg} / \mathrm{L}$, 空白组由 $0.50 \mathrm{mg} / \mathrm{L}$ 上升至 $0.65 \mathrm{mg} / \mathrm{L}$, 持 续扰动 1 周的过程中 $(14 \sim 20 \mathrm{~d})$ 各实验组下降至 $0.50 \mathrm{mg} / \mathrm{L}$ 左右. 各实验组上覆水 $\mathrm{TP}$ 浓度在实验进行前 1 周 $(0 \sim 7 \mathrm{~d})$ 由 $0.01 \mathrm{mg} / \mathrm{L}$ 最高增加至 $0.035 \mathrm{mg} / \mathrm{L}$, 空白组由 $0.01 \mathrm{mg} / \mathrm{L}$ 最高增加至 $0.055 \mathrm{mg} / \mathrm{L}$, 随后的实验 过程中 $(7 \sim 20 \mathrm{~d})$ 各实验组与空白组维持在第 1 周结束时的水平 $(8 \mathrm{~d})$.

由上述分析可知, 随着底质的沉积稳固及由于植物的生长对底质稳固作用加强, 扰动情况下上覆水 SS 浓度是呈降低趋势的. 而上覆水 $\mathrm{COD}_{\mathrm{Mn}} 、 \mathrm{TN} 、 \mathrm{TP}$ 浓度却呈增加趋势, 这是由于扰动情况下, 底质中可溶态营 养盐持续释放, 使上覆水中营养盐表现出 “积累” 的特征. 两种底质上覆水在扰动条件下受污染的程度不同, 新生底质在实验期间上覆水 SS 浓度要低于沙壤底质, 营养盐的释放量也低于沙壤底质, 尤其在没有植物稳 

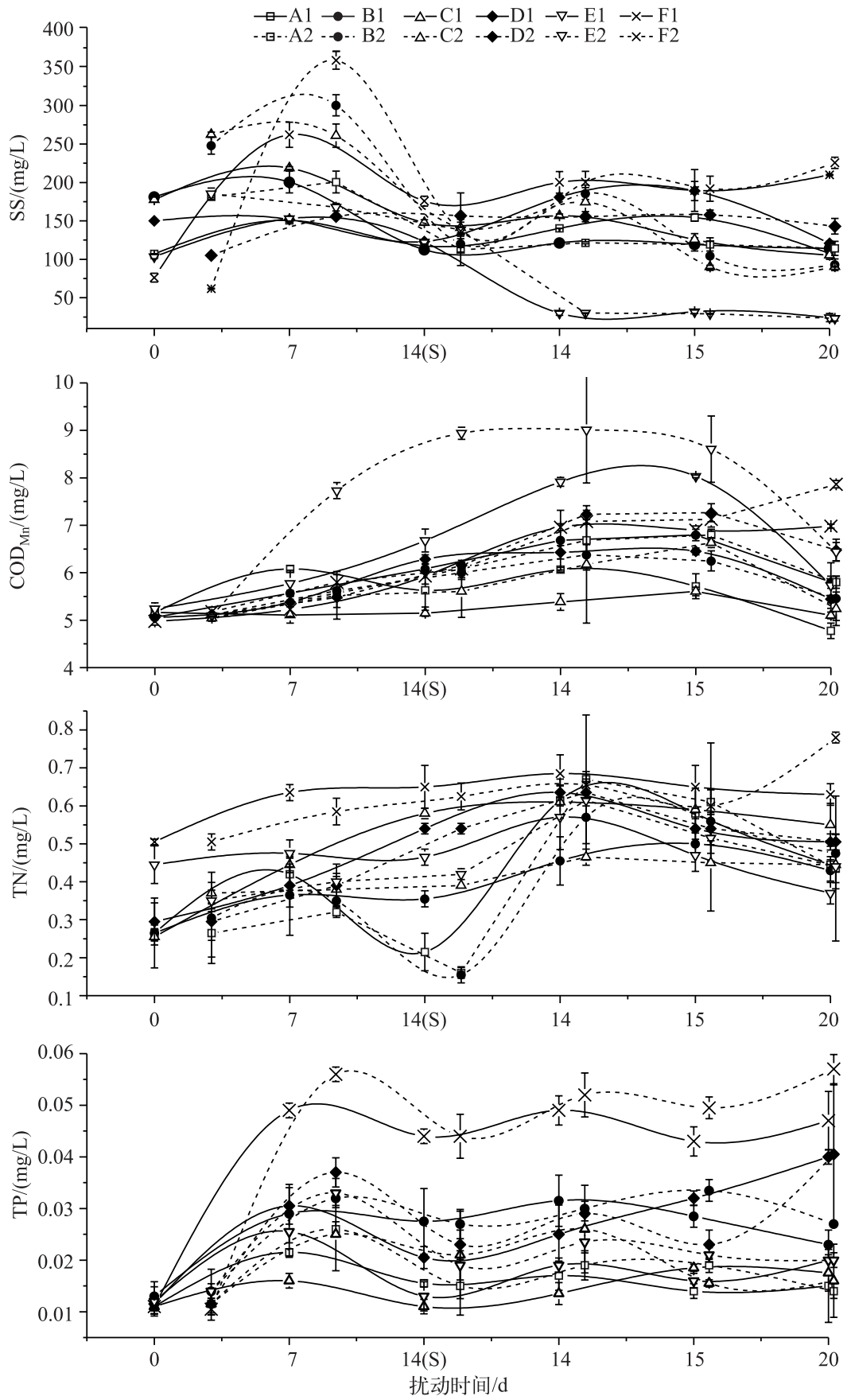

图 2 不同实验组上覆水悬浮颗粒物浓度 $(a)$ 、高锰酸盐指数 $(b)$ 、总氮浓度 $(\mathrm{c})$ 和总磷浓度 $(\mathrm{d})$ 随时间的变化

$(A 、 B 、 C 、 D 、 E$ 和 $F$ 分别代表双穗雀稗组、李氏禾组、香菇草组、黄花水龙组、黄菖蒲组和空白实验组,

下同; 1 和 2 分别表示植物种植在新生底质和沙壤底质; $\mathrm{S}$ 表示停止扰动,静止 $6 \mathrm{~h}$ )

Fig.2 Concentrations of suspended solid (a), $\mathrm{COD}_{\mathrm{Mn}}(\mathrm{b})$, total nitrogen (c) and total phosphorus $(\mathrm{d})$ of overlying water with time-variation in experimental groups 
固作用的空白组更为明显. 可见, 植物的生长可以有效减少由于扰动带来的颗粒物悬浮问题, 减少底质中营 养盐的释放带来的上覆水中营养盐浓度增加的问题.

2.1.2 上覆水水质组间差异 各实验组和空白组上覆水 SS 浓度在实验结束时 $(20 \mathrm{~d})$ 存在差异, 黄花水龙组 (D) 在各实验组中最高, 达到 $125 \mathrm{mg} / \mathrm{L}$; 黄菖蒲组 (E) 最低, 接近 $25 \mathrm{mg} / \mathrm{L}$; 双穗雀稗组 (A)、李氏禾组 $(B)$ 和 香菇草组 (C) 均达到 $100 \mathrm{mg} / \mathrm{L}$; 空白对照组则远远高于其他实验组,达到 $200 \mathrm{mg} / \mathrm{L}$. 双穗雀稗组、李氏禾组、 香菇草组和黄菖蒲组的新生底质与沙壤底质上覆水 SS 浓度在实验结束后没有显著差异, 黄花水龙组 (D) 与 空白对照组 (F) 的沙壤底质上覆水 $\mathrm{SS}$ 浓度高于新生底质, 分别为 225 与 $200 \mathrm{mg} / \mathrm{L}, 140$ 与 $120 \mathrm{mg} / \mathrm{L}$ (图 3).

各实验组与空白组上覆水 $\mathrm{COD}_{\mathrm{Mn}}$ 在实验结束时 $(20 \mathrm{~d})$ 存在差异, 同时各实验组中沙壤底质与新生底质 上覆水之间也有明显区别. 双穗雀稗组、李氏禾组、香菇草组和黄花水龙组新生底质上覆水 $\mathrm{COD}_{\mathrm{Mn}}$ 下降至 5 $\mathrm{mg} / \mathrm{L}$, 黄菖蒲组新生底质上覆水 $\mathrm{COD}_{\mathrm{Mn}}$ 超过 $6 \mathrm{mg} / \mathrm{L}$, 空白对照组上覆水 $\mathrm{COD}_{\mathrm{Mn}}$ 达到 $7 \mathrm{mg} / \mathrm{L}$. 各实验组沙壤 底质上覆水 $\mathrm{COD}_{\mathrm{Mn}}$ 要高于新生底质, 各实验组沙壤底质上覆水 $\mathrm{COD}_{\mathrm{Mn}}$ 达到 $6 \mathrm{mg} / \mathrm{L}$, 空白对照组沙壤底质上 覆水 $\mathrm{COD}_{\mathrm{Mn}}$ 达到 $7.5 \mathrm{mg} / \mathrm{L}$ (图 3 ).

各实验组沙壤底质上覆水 $\mathrm{TN}$ 浓度在实验结束时达到 $0.45 \mathrm{mg} / \mathrm{L}$, 双穗雀稗组、李氏禾组、黄菖蒲组新生 底质上覆水 $\mathrm{TN}$ 浓度为 $0.4 \mathrm{mg} / \mathrm{L}$, 香菇草组、黄花水龙组新生底质上覆水 $\mathrm{TN}$ 浓度为 $0.55 \mathrm{mg} / \mathrm{L}$; 空白对照组 沙壤底质上覆水 $\mathrm{TN}$ 浓度达到 $0.8 \mathrm{mg} / \mathrm{L}$, 新生底质上覆水 $\mathrm{TN}$ 浓度达到 $0.6 \mathrm{mg} / \mathrm{L}$ (图 3).
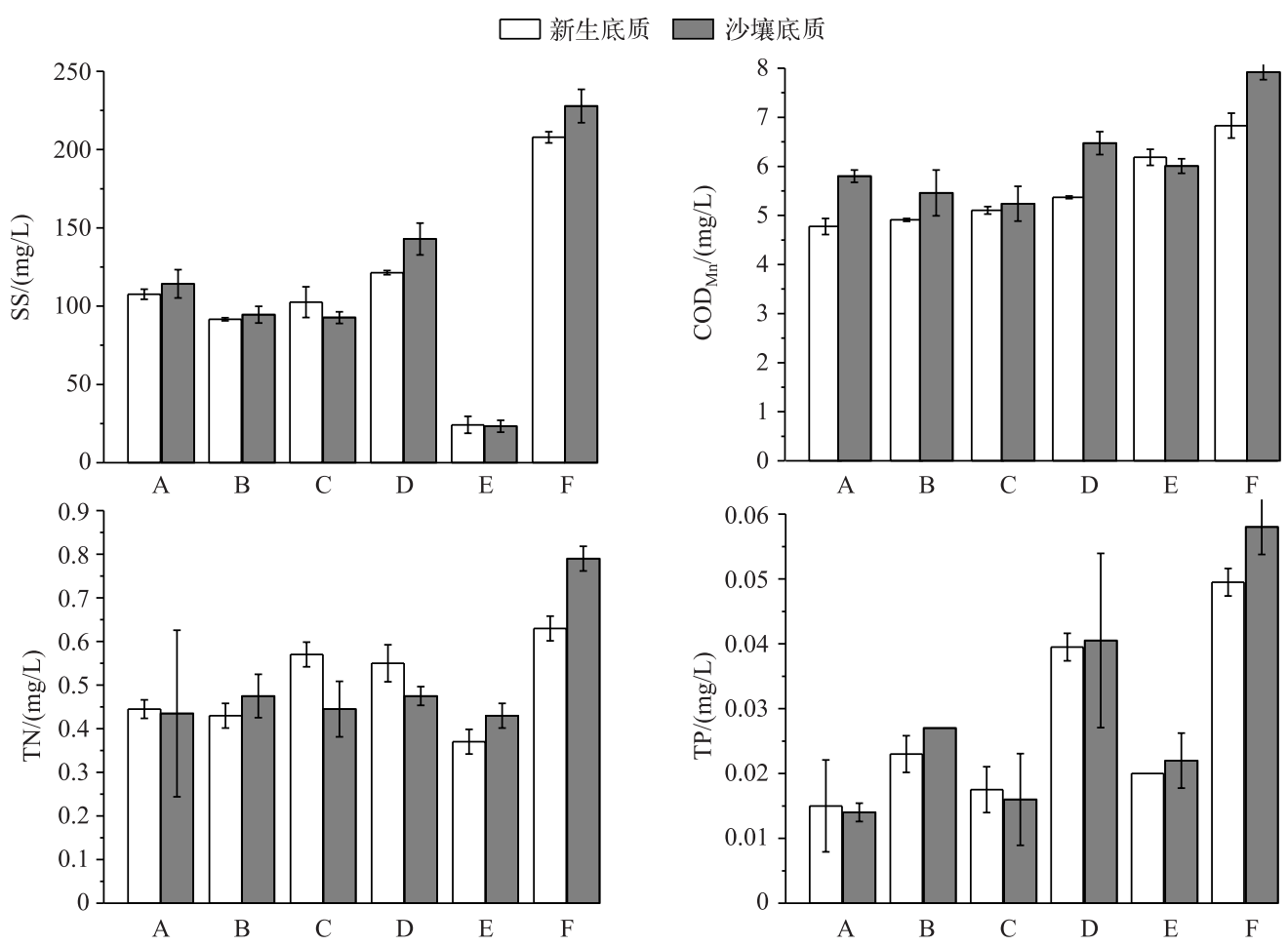

图 3 实验后各实验组上覆水指标

Fig.3 Overlying water quality of experimental groups at the end of the experiment

各实验组与空白组上覆水 TP 浓度差异明显, 双穗雀稗组、香菇草组和黄菖蒲组均在 $0.02 \mathrm{mg} / \mathrm{L}$ 以下, 李 氏禾组在 $0.025 \mathrm{mg} / \mathrm{L}$ 左右, 黄花水龙组则达到 $0.04 \mathrm{mg} / \mathrm{L}$, 空白对照组超过 $0.05 \mathrm{mg} / \mathrm{L}$, 沙壤底质与新生底质 上覆水 $\mathrm{TP}$ 浓度没有明显差异 (图 3).

可见在相同扰动条件下,空白对照组各项水质指标值均明显大于实验组, 说明不同植物的生长可不同 程度地抑制底质颗粒物悬浮和污染物释放. 不同植物对悬浮颗粒物、氮磷营养物的抑制作用差异较大, 黄菖 
蒲显示出较强的抑制作用, 其次是双穗雀稗、李氏禾、香菇草, 黄花水龙在 5 种实验植物中抑制作用最弱. 新 生底质与沙壤底质相比,其上覆水颗粒悬浮物更低,营养物质更少,在扰动下稳定性较好.

\section{2 底质稳固性指标的变化分析}

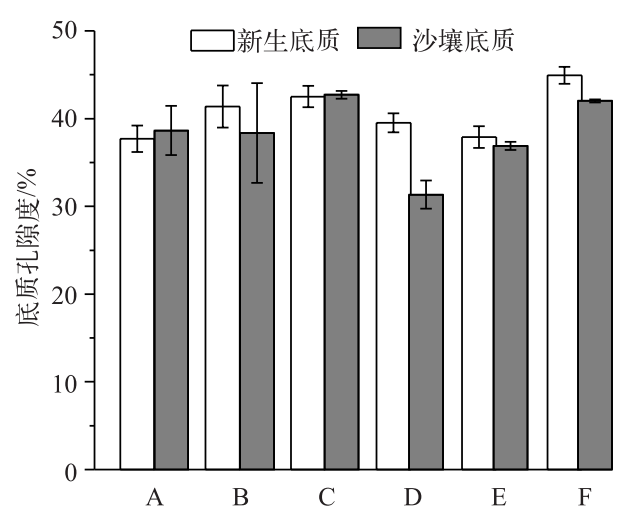

图 4 实验后各实验组底质孔隙度

Fig.4 Sediment porosity of experimental groups at the end of the experiment

2.2 .1 实验后底质孔隙度组间的差异 孔隙度反映底质孔 隙状况和松紧程度,实验前新生底质孔隙度为 $37 \%$, 沙壤 底质孔隙度为 $45 \%$, 实验后空白对照组新生底质孔隙度升 高达到 $45 \%$, 沙壤底质下降至 42\% ( 图 4). 本实验条件下, 随着实验的进行, 一方面底质自身沉积作用减小底质孔隙 度,另一方面扰动造成对表层底质颗粒结构的冲击和切 割, 使底质更加松散, 增加其空隙度. 沙壤底质对照组孔隙 度下降的原因可能是由于沙壤底质颗粒较大,实验的扰动 条件不足以对其颗粒结构产生冲击;而新生底质颗粒较 小, 在实验扰动条件下, 颗粒间分散使孔隙度升高. 实验 后, 各实验组沙壤底质与空白组沙壤底质相比, 孔隙度均 下降. 说明各沙壤底质实验组由于植物的生长, 对沙壤底 质颗粒的 “聚集” 有正向作用, 但不同植物对沙壤质颗粒的 这种正向作用差异较大 ${ }^{[14]}$. 实验结束时各实验组沙壤底质 的孔隙度均低于初始孔隙度,其中双穗雀稗、李氏禾以及 黄菖蒲作用较强,使沙壤底质孔隙度均下降至 37\%, 黄花水龙组沙壤底质孔隙度下降至 30\%, 香菇草组沙壤 底质孔隙度则没有明显变化, 达到 $43 \%$. 各实验组新生底质孔隙度均高于实验前, 达到 $40 \%$, 但小于空白新 生底质组 $45 \%$, 说明各新生底质实验组由于植物的生长, 对新生底质颗粒有一定的“聚集”作用,但这种“聚 集”作用不足以抵消扰动对颗粒物的“扩散”作用,因此孔隙度仍旧高于实验前.

2.2.2 实验后底质粒径分布变化 粒径分布是重要的底质指标, 研究表明粒径分布情况不仅决定底质的悬浮 和抗侵蚀能力, 同时也影响着底质组成物质的比例和形态 ${ }^{[15-16]}$. 实验前后各组新生底质小于 $0.05 \mathrm{~mm}$ 的粒 径比例均达到 90\%, 大于 $0.1 \mathrm{~mm}$ 的粒径比例则大于 5\% (图 5). 实验前长江中下游沙壤底质组大于 $0.1 \mathrm{~mm}$ 的粒径比例超过 $10 \%, 0.05 \sim 0.10 \mathrm{~mm}$ 的粒径比例则不到 5\%, 小于 $0.05 \mathrm{~mm}$ 的粒径比例为 $85 \%$. 双穗雀稗、 李氏禾组沙壤底质实验后小于 $0.05 \mathrm{~mm}$ 的粒径比例均提高至 $90 \%$, 大于 $0.1 \mathrm{~mm}$ 的粒径比例由 $10 \%$ 下降至 $5 \%$. 黄菖蒲组、黄花水龙组、空白对照组大于 $0.1 \mathrm{~mm}$ 的粒径比例下降, 由 $10 \%$ 下降至 5\% 左右. 香菇草组中 沙壤底质的粒径分布在实验前后没有发生明显变化, 大于 $0.1 \mathrm{~mm}$ 的粒径比例仍然较高, 超过 $10 \%$. 空白对 照组实验前后粒径分布没有显著差异; 实验前后各实验组沙壤底质粒径分布变化总体上小于 $0.05 \mathrm{~mm}$ 的比 例提高, 达到 $90 \%, 0.05 \sim 0.10 \mathrm{~mm}$ 、大于 $0.1 \mathrm{~mm}$ 的粒径比例下降; 各实验组、空白组新生底质实验后粒径分 布与实验前相比没有发生显著差异, 小于 $0.05 \mathrm{~mm}$ 的粒径比例均达到 $90 \%$, 大于 $0.1 \mathrm{~mm}$ 的粒径比例则小 于 $5 \%$.

\section{3 植物生长对底质稳固性的影响分析}

2.3.1 植物生物量与根系特征 5 种植物在实验过程中长势良好, 实验后生物量增加. 黄花水龙是浮叶植物, 实验后植物鲜重达到 $1.2 \mathrm{~kg} / \mathrm{m}^{2}$. 李氏禾与双穗雀稗均属于禾本科植物, 两者都有着发达的匍匐茎和根壮茎, 实验后李氏禾鲜重增加到 $0.85 \mathrm{~kg} / \mathrm{m}^{2}$, 双穗雀稗鲜重增加到 $0.65 \mathrm{~kg} / \mathrm{m}^{2}$. 黄菖蒲是湿生/挺水植物, 枝叶茂 盛, 根茎短粗, 环境适应性较好, 鲜重在实验前后有较快的增长, 增加至 $0.75 \mathrm{~kg} / \mathrm{m}^{2}$. 香菇草是一种低矮 (株 高 $15 \mathrm{~cm}$ ) 的蔓生挺水/湿生植物, 生物量远远小于其他较为高大的挺水/湿生植物, 实验后生物量增加至 $0.52 \mathrm{~kg} / \mathrm{m}^{2}$ (图 6).

已有研究认为根长密度、根表面积密度、根重密度是反映根系特征的重要指标, 植物根系与土壤理化性 质变化息息相关 ${ }^{[17]}$. 须根长度密度可以反映一种植物根部须根的伸展情况, 须根表面积密度可以反映植物 根部和底质的接触情况, 根重密度则直接体现须根的数量. 在选取的 5 种水生/湿生植物中, 黄菖蒲的直径 $\mathrm{d} \leqslant 1 \mathrm{~mm}$ 须根根长密度、根表面积密度较高, 而李氏禾根重密度较高, 5 种水生/湿生植物的根系参数有明显 

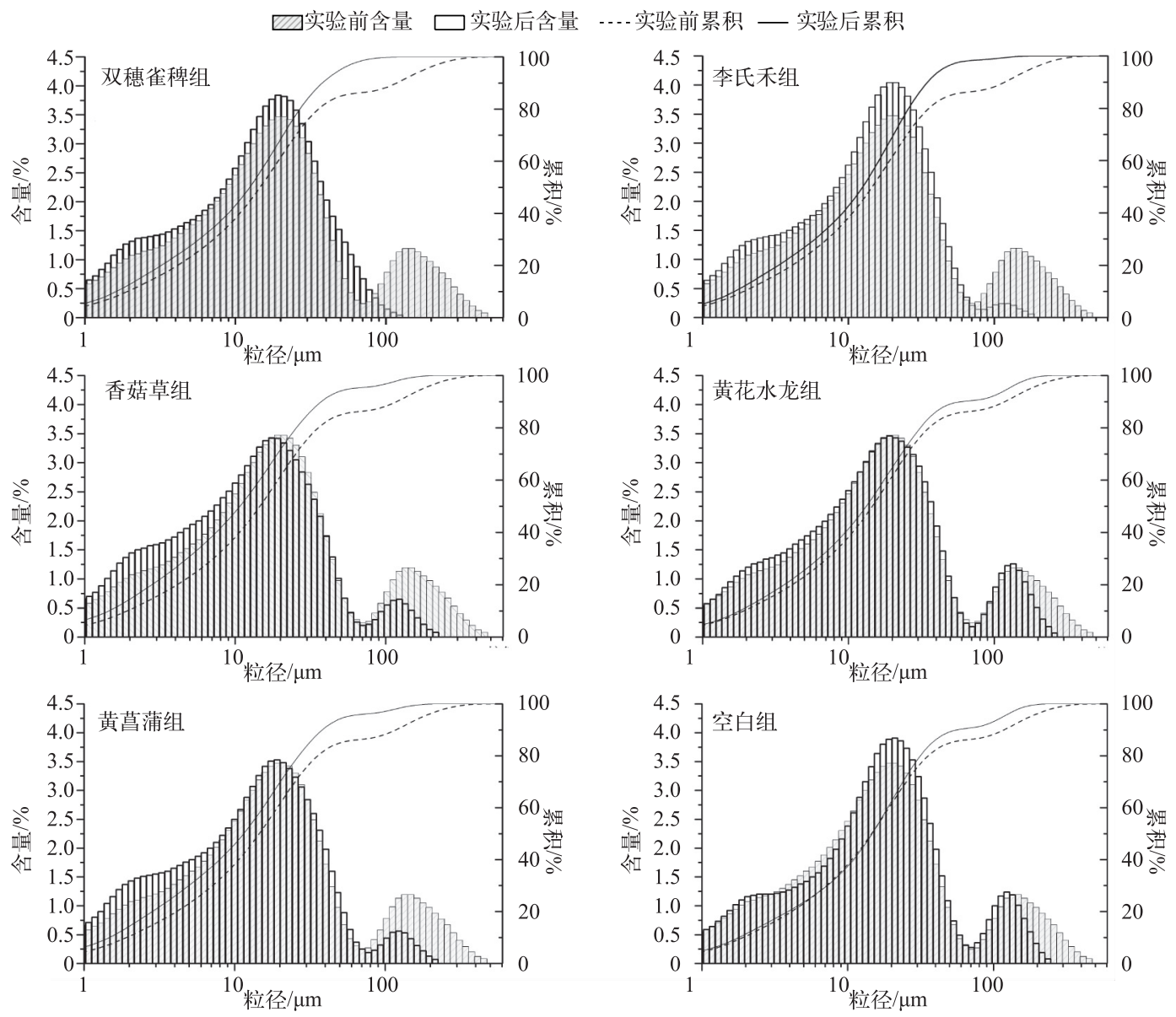

图 5 实验前后各实验组和空白组底质的粒径分布

Fig.5 Distribution of particle diameter of experimental groups before and after the experiment

的差异 (表 1 ), 但香菇草与黄菖蒲一样有很强的适应 性, 可以在实验装置中快速扎根稳定.

$\mathrm{d} \leqslant 1 \mathrm{~mm}$ 的须根特征参数之间有密切的联系, 根 长密度与根表面积密度之间的相关系数达到 0.9939 , 根重密度与二者之间的相关系数也达到了 0.4163 . 从 拟合的函数上看, 3 个参数之间两两呈正相关 (图 7), 即说明植物须根量 (根重密度) 越大其影响作用范围越 大, 与底质接触越密切.

2.3.2 植物生长对底质稳固性的影响分析底质稳固 性能与底质的粒径分布、孔隙度以及底质的化学特征 有密切联系, 而 $\mathrm{d} \leqslant 1 \mathrm{~mm}$ 的须根对底质的物理、化学特 征有着直接的影响 ${ }^{[18-19]}$. 根长密度和根表面积密度与 底质孔隙度有着显著的负相关性 (表 2). 须根紧实底 质,减少孔隙度,增强了底质抗扰动性能. 不同粒径级别数量间的相关关系指出了它们之间此消彼长的数量 关系, 底质粒径分布研究也指出了实验后粒径 $<50 \mu \mathrm{m}$ 底质的增加及其他粒径级别的减少. 根长密度、根表

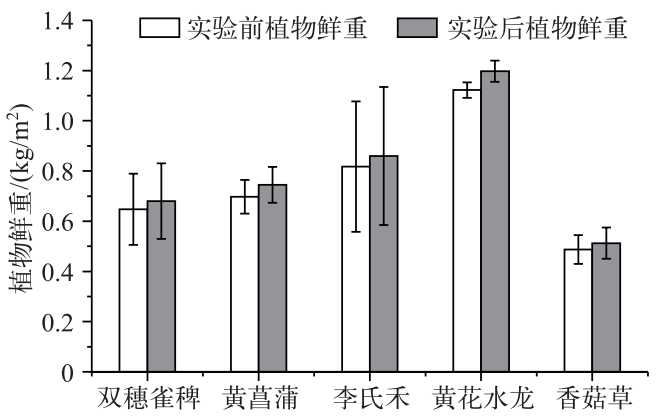

图 6 不同实验组实验前后植物生物量变化

Fig.6 Plant biomass of experimental groups before and after the experiment 
表 15 种湖滨带植物须根 $(\mathrm{d} \leqslant 1 \mathrm{~mm})$ 的特征参数

Tab.1 Characteristic parameters of the fine root $(d \leqslant 1 \mathrm{~mm})$ of 5 littoral zone plants

\begin{tabular}{lccc}
\hline 植物种类 & 根长密度 $/\left(\mathrm{cm} / \mathrm{cm}^{3}\right)$ & 根表面积密度 $/\left(\mathrm{cm}^{2} / \mathrm{cm}^{3}\right)$ & 根重密度 $/\left(\mathrm{mg} / \mathrm{cm}^{3}\right)$ \\
\hline 双穗雀稗 & $1.057 \pm 0.245$ & $0.033 \pm 0.007$ & $0.678 \pm 0.229$ \\
黄菖蒲 & $11.495 \pm 1.188$ & $0.368 \pm 0.034$ & $1.844 \pm 0.467$ \\
李氏禾 & $7.368 \pm 0.295$ & $0.228 \pm 0.009$ & $2.537 \pm 0.361$ \\
黄花水龙 & $9.475 \pm 1.003$ & $0.294 \pm 0.058$ & $0.944 \pm 0.210$ \\
香菇草 & $0.651 \pm 0.108$ & $0.022 \pm 0.004$ & $0.16 \pm 0.008$ \\
\hline
\end{tabular}
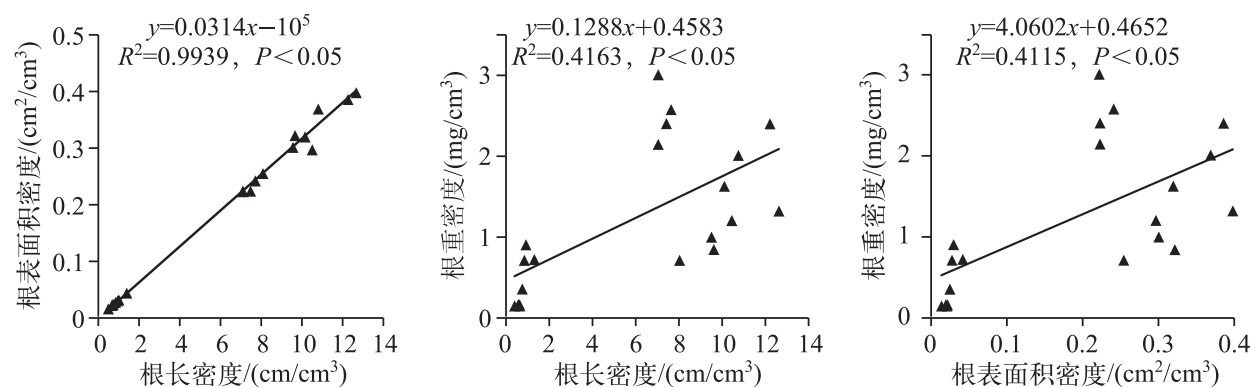

图 7 植物须根 $(\mathrm{d} \leqslant 1 \mathrm{~mm})$ 各特征参数之间的关系

Fig.7 Correlation coefficients among fine $\operatorname{root}(\mathrm{d} \leqslant 1 \mathrm{~mm})$ characteristic parameters

面积密度、根重密度与粒径 $<50 \mu \mathrm{m}$ 的底质数量有显著的正相关关系 $(P<0.05$, 表 3), 而生物量与底质的孔 隙度和粒径分布则没有显著的相关关系 (表 2). 鲁素芸指出部分水生植物根系通过穿插和分泌糖类、有机 酸甚至腐败分解等方式促进底质在水体冲击下的稳定 ${ }^{[20]}$. 植物根系不仅通过分泌活性酶、植物激素来包裹 固结底质, 其自身生长对底质的穿插压实也让底质的颗粒越来越细密, 空隙越来越少 ${ }^{[21]}$. 植物须根在底质 中的生长影响着底质的孔隙度和粒径分布, 改变了底质抵抗水体扰动的性能. 须根通过分泌有机物质包裹 粘连底质细小颗粒, 同时须根对底质的穿插挤压也使底质颗粒物质变得更加细小, 更易与须根紧密粘连.

表 2 植物生物量、 $\mathrm{d} \leqslant 1 \mathrm{~mm}$ 的须根特征参数与底质孔隙度的相关矩阵

Tab.2 Correlation matrix of plant biomass, fine root $(\mathrm{d} \leqslant 1 \mathrm{~mm})$ characteristic parameters and sediment porosity

\begin{tabular}{|c|c|c|c|c|c|}
\hline & 根长密度 & 根重密度 & 根表面积密度 & 植物生物量 & 底质孔隙度 \\
\hline 根长密度 & 1 & & & & \\
\hline 根表面积密度 & $0.700^{* * *}$ & 1 & & & \\
\hline 根重密度 & $1.000^{* * *}$ & $0.700^{\text {*** }}$ & 1 & & \\
\hline 植物生物量 & 0.102 & -0.114 & 0.101 & 1 & \\
\hline 底质孔隙度 & $-0.505^{*}$ & -0.320 & $-0.505^{*}$ & 0.362 & 1 \\
\hline
\end{tabular}

* 表示 $P<0.05$; ** 表示 $P<0.01$.

2.3.3 植物根系对底质稳固性的增益作用 底质孔隙度、底质粒径分布是上覆水水质在本次实验条件下变化 的内因, 这两项底质指标可以衡量底质稳定性能, 其改善程度用空白实验组各项参数与各个植物实验组参 数的相对差值来反映. 将这种相对差值定义为底质稳定性增益值, 分别为孔隙度增益值 $I_{1}$ 和粒径增益值 $I_{2}$. 结果 (表 4) 表明双穗雀稗、李氏禾、香菇草 3 种植物 $\mathrm{d} \leqslant 1 \mathrm{~mm}$ 的须根量 $R_{\mathrm{w}}$ 、须根长度 $R_{\mathrm{L}}$ 、须根面积 $R_{\mathrm{S}}$ 与各增 益值之间存在线型正相关关系 (斜率为 $0.006 \sim 1.727$, 黄花水龙和黄菖蒲须根参数与底质粒径分布增益值之 间存在线性负相关关系 (斜率为 $-0.091 \sim-0.011$ ). 以李氏禾须根量回归方程 $I_{1}=0.018 R_{\mathrm{W}}-0.015$ 和 $I_{2}=$ $0.025 R_{\mathrm{W}}+0.103$ 为例, 其须根量每增加 $1 \mathrm{mg} / \mathrm{cm}^{3}$, 孔隙度增加 $1.8 \%$, 粒径 $<50 \mu \mathrm{m}$ 的底质增加 $2.5 \%$. 
表 3 植物生物量、 $\mathrm{d} \leqslant 1 \mathrm{~mm}$ 的须根特征参数与底质粒径分布的相关矩阵

Tab.3 Correlation matrix of plant biomass, fine root $(d \leqslant 1 \mathrm{~mm})$ characteristic parameters and particle diameter

\begin{tabular}{|c|c|c|c|c|c|c|c|c|}
\hline & 根长密度 & 根重密度 & 根表面积密度 & 直物生物量 & D50 & D100 & D300 & D500 \\
\hline 根长密度 & 1 & & & & & & & \\
\hline 根表面积密 & $0.700^{* *}$ & 1 & & & & & & \\
\hline 根重密度 & $1.000^{* *}$ & $0.700^{* *}$ & 1 & & & & & \\
\hline 植物生物量 & 0.102 & -0.114 & 0.101 & 1 & & & & \\
\hline D50 & $0.126^{*}$ & $0.369^{*}$ & $0.126^{*}$ & -0.035 & 1 & & & \\
\hline D100 & $-0.152 *$ & $-0.302 *$ & $-0.152 *$ & 0.139 * & $-0.759^{* *}$ & 1 & & \\
\hline D300 & 0.039 & -0.079 & 0.040 & -0.130 & $-0.451^{*}$ & $-0.226^{*}$ & 1 & \\
\hline D500 & -0.065 & $-0.306^{*}$ & -0.065 & -0.118 & $-0.274^{*}$ & $-0.190^{*}$ & $0.516^{*}$ & 1 \\
\hline
\end{tabular}

*表示 $P<0.05$; ** 表示 $P<0.01$, D50、D100、D300 和 D500 分别表示底质中粒径 $\leqslant 50 、 100 、 300 、 500 \mu \mathrm{m}$ 的比例.

须根参数与改善值之间的增益值是植物根系对底质增益效果的体现, 增益值越大说明根系增益效果越 高效, 根系生长对底质增益效果的提升越明显. 双穗雀稗、李氏禾、香菇草的根系参数越大, 其生长环境中底 质的物理特征就越利于底质的稳定; 黄花水龙和黄菖蒲须根参数与底质粒径分布增益值之间存在截距大于 0 的负线性关系, 这表明随着须根生长, 根系对底质稳定的促进作用就在减弱. 这两种植物根系在须根还不 是很发达的时候, 有着不错的增益效果. 如黄花水龙根长密度与粒径分布增益回归方程的截距达到 0.933 , 当黄花水龙生长初期须根长度较小的时候, 根长密度每增加 $1 \mathrm{~cm} / \mathrm{cm}^{3}$, 粒径 $<50 \mu \mathrm{m}$ 的底质就增加 $90 \%$ 左右.

表 4 植物根系特征参数( 根长密度 (RLD)、根表面积密度 (RSAD)、根重密度 (RWD))

与底质稳固性的增益系数方程 $(P<0.01)$

Tab.4 Regression equations between sediment stabilization enhancement coefficients and fine root characteristic parameters ( root length density, root surface area density and root weight density) $(P<0.01)$

\begin{tabular}{lclll}
\hline 植物类型 & 修复项目 & 增益值与 RLD 的回归方程 & 增益值与 $\mathrm{RWD}$ 的回归方程 & 增益值与 RSAD 的回归方程 \\
\hline 双穗雀稗 & $I_{1}$ & $I_{1}=0.054 R_{\mathrm{L}}-0.002$ & $I_{1}=0.094 R_{\mathrm{W}}-0.008$ & $I_{1}=1.727 R_{\mathrm{S}}-0.002$ \\
& & $r=0.5200$ & $r=0.6617$ & $r=0.4078$ \\
& $I_{2}$ & $I_{2}=0.144 R_{\mathrm{L}}+0.016$ & $I_{2}=0.059 R_{\mathrm{W}}+0.129$ & $I_{2}=0.059 R_{\mathrm{S}}+0.129$ \\
& & $r=0.5739$ & $r=0.5115$ & $r=0.5205$ \\
李氏禾 & $I_{1}$ & $I_{1}=0.050 R_{\mathrm{L}}-0.005$ & $I_{1}=0.018 R_{\mathrm{W}}-0.015$ & $I_{1}=0.155 R_{\mathrm{S}}-0.005$ \\
& & $r=0.6259$ & $r=0.6157$ & $r=0.4259$ \\
& $I_{2}$ & $I_{2}=0.011 R_{\mathrm{L}}+0.093$ & $I_{2}=0.025 R_{\mathrm{W}}+0.103$ & $I_{2}=0.341 R_{\mathrm{S}}+0.093$ \\
& & $r=0.6967$ & $r=0.6145$ & $r=0.6967$ \\
香菇草 & $I_{1}$ & $I_{1}=0.149 R_{\mathrm{L}}-0.087$ & $I_{1}=0.012 R_{\mathrm{W}}+0.199$ & $I_{1}=0.476 R_{\mathrm{S}}-0.087$ \\
& & $r=0.6659$ & $r=0.500$ & $r=0.6659$ \\
& $I_{2}$ & $I_{2}=0.051 R_{\mathrm{L}}-0.165$ & $I_{2}=0.006 R_{\mathrm{W}}-0.133$ & $I_{2}=1.631 R_{\mathrm{S}}-0.165$ \\
& & $r=0.5779$ & $r=0.500$ & $r=0.5779$ \\
黄花水龙 & $I_{1}$ & $I_{1}=0.027 R_{\mathrm{L}}-0.162$ & $I_{1}=0.131 R_{\mathrm{W}}-0.039$ & $I_{1}=0.847 R_{\mathrm{S}}-0.162$ \\
& & $r=0.999$ & $r=0.9976$ & $r=0.999$ \\
& $I_{2}$ & $I_{2}=-0.091 R_{\mathrm{L}}+0.933$ & $I_{2}=-0.044 R_{\mathrm{W}}+0.506$ & $I_{2}=-0.029 R_{\mathrm{S}}+0.933$ \\
& & $r=0.922$ & $r=0.910$ & $r=0.922$ \\
& $I_{1}$ & $I_{1}=-0.011 R_{\mathrm{L}}+0.194$ & $I_{1}=-0.025 R_{\mathrm{W}}+0.113$ & $I_{1}=-0.344 R_{\mathrm{S}}+0.194$ \\
& & $r=0.745$ & $r=0.7342$ & $r=0.7449$ \\
黄菖蒲 & $I_{2}=-0.025 R_{\mathrm{L}}+0.498$ & $I_{2}=-0.015 R_{\mathrm{W}}+0.491$ & $I_{2}=-0.079 R_{\mathrm{S}}+0.498$ \\
& & $r=0.3639$ & $r=0.9615$ & $r=0.3639$ \\
& & & &
\end{tabular}


植物须根的数量和质量也是影响底质稳定性的重要因素, 黄菖蒲与黄花水龙的根长密度分别为 11.495 和 $9.475 \mathrm{~cm} / \mathrm{cm}^{3}$, 根表面积密度分别为 0.368 和 $0.294 \mathrm{~cm}^{2} / \mathrm{cm}^{3}$, 根重密度为 1.844 和 $0.944 \mathrm{mg} / \mathrm{cm}^{3}$, 两种植 物根系参数对底质孔隙度增益值分别为 $15 \%$ 和 $9 \%$, 两种植物根系参数对底质粒径分布增益值分别为 $92 \%$ 和 47\% ; 双穗雀稗、李氏禾和香菇草的根长密度分别为 1.057 、. 368 和 $0.651 \mathrm{~cm} / \mathrm{cm}^{3}$, 根表面积密度分别为 $0.033 、 0.228$ 和 $0.022 \mathrm{~cm}^{2} / \mathrm{cm}^{3}$, 根重密度为 $0.678 、 2.537$ 和 $0.160 \mathrm{mg} / \mathrm{cm}^{3}, 3$ 种植物根系参数对底质孔隙度的 增益值分别为 $6 \%$ 、36\%和 1\%,3 种植物根系参数对底质粒径分布增益值分别为 $16 \% 、 17 \%$ 和 $-13 \%$. 不同植 物根系对底质增益系数的差异来自于根系分泌的活性酶、植物激素的种类以及部分根系死亡分解时分解物 质的种类性质 ${ }^{[22]} .5$ 种水生/湿生植物根系均可以对湖滨底质稳定性有改善作用, 增强底质的抗水体扰动能 力, 控制底质中营养物质的释放, 改善上覆水水质; 从效能上表现为李氏禾 $>$ 双穗雀稗 $>$ 黄菖蒲 $>$ 黄花水龙 $>$ 香菇草.

\section{3 结论}

1) 植物根系对底质稳定性增益一方面通过直径 $<1 \mathrm{~mm}$ 的须根穿插紧实底质, 造成底质孔隙度下降; 同 时须根的生长对底质的穿插挤压造成粒径 $<50 \mu \mathrm{m}$ 的颗粒增多, 这些细小颗粒更易与须根紧密粘连, 增强底 质的稳固性.

2) 直径 $\leqslant 1 \mathrm{~mm}$ 的须根量 $R_{\mathrm{w}}$ 、须根长度 $R_{\mathrm{L}}$ 、须根面积 $R_{\mathrm{S}}$ 与底质孔隙度和粒径分布增益值之间存在线性 回归关系; 双穗雀稗、李氏禾、香菇草的须根量 $R_{\mathrm{w}}$ 、须根长度 $R_{\mathrm{L}}$ 、须根面积 $R_{\mathrm{S}}$ 与粒径分布、孔隙度增益值之间 存在斜率为 0.006 1.727 的线型正相关关系; 黄花水龙和黄菖蒲的须根参数与粒径分布增益值之间存在斜 率为 $-0.091 \sim-0.011$ 的线性负相关关系. 双穗雀稗、李氏禾、香菇草的根系参数越大, 其生长环境中底质的 物理特征就越利于底质的稳固; 黄菖蒲、黄花水龙则随着根系参数的升高, 对底质稳固的增益作用减小.

3) 黄菖蒲与黄花水龙具有发达的植物根系, 两种植物根系参数对底质孔隙度增益值分别为 $15 \%$ 和 $9 \%$, 对底质粒径分布增益值分别为 $92 \%$ 和 $47 \%$; 双穗雀稗、李氏禾、香菇草根系参数较小, 3 种植物根系参 数对底质孔隙度的增益值分别为 $6 \%$ 、36\%和 $1 \%$, 对底质粒径分布增益值分别为 $16 \% 、 17 \%$ 和 $-13 \%$.

综上所述, 5 种植物通过根系提高底质的稳定性, 减少底质在水力扰动下悬浮物质以及营养盐的释放. 从效能上表现为李氏禾 > 双穗雀稗 > 黄菖蒲 > 黄花水龙 >香菇草.

\section{4 参考文献}

[ 1 ] Lu Hongwei, Zeng Guangming, Jin Xiangcan. Research progress on lake-side ecological restoration and rebuilding. Journal of Hunan University (Natural Science)，2003，(S1) : 85-89. [ 卢宏玮, 曾光明, 金相灿. 湖滨生态带恢复与重建研究 进展. 湖南大学学报: 自然科学版, 2003, (S1) : 85-89.]

[ 2 ] Hu Xiaozhen, Xu Qiujin, Jin Xiangcan et al. Summary on the interaction between sediment and aquatic macrophyte. Journal of Biology, 2011, 28(2):73-76. [ 胡小贞, 许秋瑾, 金相灿等. 湖泊底质与水生植物相互作用综述. 生物学杂 志, $2011,28(2): 73-76$.

[ 3 ] Zhu Xianmo, Tian Jiying. The study on strengthening anti-scourability and penetrability of soil in loss plateau. Journal of Soil and Water Conservation, 1993, 7(3): 1-10. [朱显谟, 田积荣. 强化黄土高原土壤渗透性及抗冲性的研究. 水土 保持研究, 1993, 7(3): 1-10.]

[ 4 ] Wu Yan, Liu Shiquan, Wang Jinxi. Effect of plant root system on soil anti-erosion. Chinese Journal of Applied And Environmental Biology, 1997, (2) : 4. [吴彦, 刘世全, 王金锡. 植物根系对土壤抗侵蚀能力的影响. 应用于环境生物学 报, 1997, (2): 4.]

[ 5 ] Horppila J, Nurminen L. Effects of different macrophyte growth forms on sediment and P resuspension in a shallow lake. Hydrobiologia, 2005, 545(1): 167-175.

[ 6 ] Charles DD. The importance of emergent vegetation in reducing sediment resuspension in wetlands. Journal of Freshwater Ecology, 1990, 5(4) : 467-473.

[ 7 ] Horppila J, Nurminen L. Effects of submerged macrophytes on sediment resuspension and internal phosphorus loading in Lake Hiidenvesi (southern Finland). Water Research, 2003, 37(18) : 4468-4474.

[ 8 ] Wu Qingxin, Liu Jie, You Shaohong et al. Decontamination and mechanism of $\operatorname{Cr}$ ( VI ) -polluted water in constructed wet- 
land planted with Lessrsia hexandra Swartz. Acta Scientiae Circumstantiae, 2014, 34(9): 2306-2312. [伍清新, 刘杰, 游 少鸿等. 李氏禾湿地系统净化 $\operatorname{Cr}(\mathrm{VI})$ 污染水体的机理研究. 环境科学学报, 2014, 34(9) :2306-2312.]

[ 9 ] Zhu Yuhui. Plant screening and the purification efficiency for eutrophic wastewater in constructed wetland[Dissertation]. Hangzhou: Zhejiang University, 2008. [ 祝宇慧. 人工湿地植物笁选及其对营养型污水的净化效果研究 [ 学位论文]. 杭州: 浙江大学, 2008.]

[10] You Chaoyang, Wan Yulong, Han Sujuan et al. Microbial distribution in constructed wetland of Iris pseudacorus L. Journal of Anhui Agri Sci, 2010, 11(9) : 26-28. [ 尤朝阳, 万玉龙, 韩苏娟等. 黄菖蒲人工湿地微生物分布特点研究. 安徽 农业科学, 2010, 11(9): 26-28.]

[11] Han Sujuan, You Chaoyang, Wan Yulong et al. Characteristic comparison of six aquatic plants in constructed wetlands. Journal of Anhui Agri Sci, 2010, 38(12) : 6367-6368. [ 韩苏娟, 尤朝阳, 万玉龙等. 6 种水生植物在人工湿地中特性 的比较. 安徽农业科学, 2010, 38(12):6367-6368.]

[12] Wang Chao, Zhang Wenming, Wang Peifang et al. Removal of nitrogen and phosphorus in eutrophic water by Jussiaea stipulacea Ohwi. Environmental Science, 2007, 28(5) : 975-981. [王超, 张文明, 王沛芳等. 黄花水龙对富营养化水体中 氮磷去除效果的研究. 环境科学, 2007, 28(5) :975-981.]

[13] Jiang Xia ed. Handbook of investigation and assessment of sediment quality. Beijing: Science Press, 2012.[姜霞. 沉积物 质量调查评估手册. 北京: 科学出版社, 2012.]

[14] He Xiaoyan. The effect of the physical properties of the sediments on contaminant adsorption/desorption and interfacial diffusion[Dissertation]. Shanghai: Shanghai University, 2012. [ 贺晓艳. 底泥物理特性对污染物吸附/解吸和界面扩散 的影响 [学位论文]. 上海: 上海大学, 2012.]

[15] Li Zhanhai, Chen Shenliang, Zhang Guoan. The component of sand and characteristic of resuspension in the water of Chongming east beach of Yangtze River Estuary. Acta Oceanologica Sinica, 2008, 30(6):154-163. [ 李占海, 陈沈良, 张 国安. 长江口崇明东滩水域悬沙粒径组成和再悬浮作用特征. 海洋学报: 中文版, 2008, 30(6) : 154-163.]

[16] Mahesh BS, Warrier AK, Mohan R et al. Response of Long Lake sediments to Antarctic climate: A perspective gained from sedimentary organic geochemistry and particle size analysis. Polar Science, 2015, 9(4) : 359-367.

[17] Zhang Liangde, Xu Xuexuan, Hu Wei et al. Spatial distribution of fine roots of a Robiuia pseudoacacia plantation in Yangou Watershed in the hilly region of the loess plateau. Scientia Silvae Sinicae, 2011, 47(11): 31-36. [张良德, 徐学 选，胡伟等. 黄土丘陵区燕沟流域人工刺槐林的细根空间分布特征. 林业科学, 2011, 47(11): 31-36.]

[18] Ding Qingzhang, Liu Xueqin, Zhang Xiaoke. Impacts of water level fluctuations on substrate environments of lakeshore zone of the lakes in the middle and lower reaches of the Yangtze River. J Lake Sci, 2014, 26(3) :340-348. DOI: 10. 18307/2014.0302. [丁庆章, 刘学勤, 张晓可. 水位波动对长江中下游湖泊湖滨带底质环境的影响. 湖泊科学, $2014,26(3): 340-348$. ]

[19] Lin Jianhua, Yang Yang, Li Li et al. Characteristics of growth and radial oxygen loss of eight wetland plants. J Lake Sci, 2015, 27 (6) : 1042-1048. DOI: 10.18307/2015.0608. [林剑华, 杨扬, 李丽等. 8 种湿地植物的生长状况及泌氧能 力. 湖泊科学, $2015,27(6): 1042-1048$. ]

[20] Lu Suyun ed. Plant root system ecology and root disease of biological control. Beijing: China Renmin University Press, 1990. [ 鲁素芸. 植物根际生态学与病根生物防治进展. 北京: 中国人民大学出版社, 1990.]

[21] Wang Ku. Effects of plant roots on soil anti-erosion. Soil and Environmental Science, 2001, 10(3): 250-252. [王库. 植物 根系对土壤抗侵蚀能力的影响. 土壤与环境, 2001, 10(3): 250-252.]

[22] Luo Yongqing, Zhao Xueyong, Li Meixia. Ecological effect of plant root exudates and related affecting factors: A review. Chinese Journal of Applied Ecology, 2012, 23(12):3496-3504. [罗永清, 赵学勇, 李美霞. 植物根系分泌物生态效应 及其影响因素研究综述. 应用生态学报, 2012, 23(12):3496-3504.] 